\title{
Synthesis, Characterization and Application of Chitosan-Coated Magnetite Nanoparticles in Drug Delivery
}

\author{
D.Balaji, M.Vijay Albert William, C.Sarala Rubi
}

\begin{abstract}
Magnetite nanoparticles were used extensively for various applications. In the present study, magnetite nanoparticles were synthesized and characterized by atomic force microscopy (AFM). AFM images showed that the obtained particles were perfectly spherical. Functionality is afforded to these magnetite nanoparticles by adding biocompatible polymer chitosan during the synthesis. AFM phase image clearly showed that the magnetite core is encapsulated with the polymeric shell. Fourier-transform infrared spectroscopy (FTIR) study showed the chitosan absorption on $\mathrm{Fe}_{2} \mathrm{O}_{3}$ nanoparticle surface. The drug sulphamethoxazole was loaded over magnetite nanoparticles and the encapsulation efficiency of drug was calculated at different concentrations of magnetite. The encapsulation efficiency increases with increase in the concentration of magnetite. Thus, an attempt was made in synthesizing drug loaded biopolymer magnetite nanoparticles suitable for targeted drug delivery.
\end{abstract}

Keywords: Nanoparticle: Chitosan; AFM; Coating; Polymer

\section{INTRODUCTION}

Magnetic oxide nanoparticles were extensively investigated due to their vast applications. Due to their better magnetic properties and lower toxicity, magnetite nanoparticles are mostly preferred for the drug delivery system. Magnetite nanoparticles are widely used in the biomedical applications because of their smaller size, larger surface, and enhanced solubility. The biomedical applications of magnetite nanoparticles include: drug delivery, chemotherapy, magnetofection, hyperthermia, scaffold-based tissue engineering, MRI contrast agents, organ/tissue imaging, theranostic platforms, in vitro bioseparation, bioanalysis, and immunoassays [1-3]. Due to the multifunctional properties of the magnetite nanoparticles, they are able to interact with complex cellular functions. This rapidly growing field requires cross-disciplinary research and

Revised Manuscript Received on February 05, 2020.

* Correspondence Author

D.Balaji*, Department of Electronics \& Communication Engineering, Vel Tech Rangarajan Dr. Sagunthala R \& D Institute of Science and Technology, Chennai, Tamil Nadu, India. Email: balatec@gmail.com

M.Vijay Albert William, Department of Electronics \& Communication Engineering, Vel Tech Rangarajan Dr. Sagunthala R \& D Institute of Science and Technology, Chennai, Tamil Nadu, India. Email: vijayalbert007@gmail.com

C.Sarala Rubi, Department of Physics, Vel Tech Rangarajan Dr. Sagunthala R \& D Institute of Science and Technology, Chennai, Tamil Nadu, India. Email: sarala.rubi@yahoo.com

(C) The Authors. Published by Blue Eyes Intelligence Engineering and Sciences Publication (BEIESP). This is an open access article under the CC BY-NC-ND license (http://creativecommons.org/licenses/by-nc-nd/4.0/) provides opportunities to develop multifunctional devices to treat cancer. In order to reduce drug doses, coats associated with drug treatment, and potential side effects, the magnetite nanoparticles are attached to the drugs. Generally, oxides of iron are short blood half-life and their primary application of oxides of iron is for imaging of liver. However, the surface modification of nanoprticles leads to long blood circulation times. Hence, the surface modified nanoparticles are highly useful for imaging of the vascular compartment and specific target. The syntheses of uniform iron nanoparticles are reported very little. The synthesis of iron nanoparticles by sonochemical decomposition of iron pentacarbonyl in the presence of polyvinylpyrrolidone (PVP) has been reported. The sonochemical decomposition method was used to synthesize various nanostructured materials. The inability to control particle size is one of the drawbacks in the synthesis of nanoparticles by sonochemical decomposition method [4-6]. The thermal decomposition method was also recently used to synthesize iron oxide nanoparticles from iron pentacarbonyl in the presence of oleic acid at $100{ }^{\circ} \mathrm{C}$ [7-11]. Monosized iron nanoparticles were also synthesized at 300 ${ }^{\circ} \mathrm{C}$ from iron-oleic acid metal complex. Magnetite nanoparticles are particularly desirable because it displays a strong magnetic behavior, and is less sensitive to oxidation than magnetic transition metals such as cobalt, iron, and nickel. For in-vivo applications, it is important that well-defined organic coatings surround the magnetite particles. It is rationalized that this will prevent any aggregation of the nanoparticles in-vivo, and may also enable efficient excretion and protection of the body from toxicity [12-16]. In this work, it is aimed to develop drug loaded biopolymer magnetite nanoparticles for biomedical application. The main objective is to synthesize and characterize magnetite $\left(\mathrm{Fe}_{3} \mathrm{O}_{4}\right)$ nanoparticles and to afford functionality to the $\mathrm{Fe}_{3} \mathrm{O}_{4}$ nanoparticles by addition of biocompatible polymer chitosan. It is also aimed to load the drug sulphamethoxazole to the magnetite nanoparticles and to estimate the encapsulation efficiency of drug at different concentrations of magnetite.

\section{EXPERIMENTAL METHODS}

\subsection{Materials}

Ferric chloride $\left(\mathrm{FeCl}_{3} .6 \mathrm{H}_{2} \mathrm{O}, 98 \%\right)$, Ferrous sulphate ( $\mathrm{FeSO}_{4}, 98 \%$ ), $\mathrm{NH}_{4} \mathrm{OH}, \mathrm{CH}_{3} \mathrm{COOH}$ were obtained from Sigma Aldrich. Ethyl alcohol was purchased from Hong yang corpn, China. Chitosan, less molecular weight $(<6000 \mathrm{Mn})$, was obtained from Sigma Aldrich. 
Sulphamethoxazole was purchased from Hi Media Laboratories, Mumbai. Analytical grade reagents were used without any purification.

\subsection{Synthesis of Magnetite Nanoparticles}

$0.1 \mathrm{M} \mathrm{KOH}$ solution was mixed with the solution of 0.05 $\mathrm{M} \mathrm{FeSO}_{4}$ in a three neck flask. $0.2 \mathrm{M}$ of $\mathrm{KNO}_{3}$ was added into the solution and the solution mixture was kept at $90^{\circ} \mathrm{C}$ for $24 \mathrm{~h}$ with constant stirring. Ethanol and deionized water were used to wash the resultant black precipitate. Then, the dispersion of nanoparticles took place in ethyl alcohol.

\subsection{Coating of Chitosan over Magnetite Nanoparticles}

Chitosan solution was prepared by dissolving chitosan in $1 \%$ acetic acid. $\mathrm{Fe}_{3} \mathrm{O}_{4}$ nanoparticles were purified by centrifugation before adding to chitosan solution $(0.4 \mathrm{mg} / \mathrm{ml})$. Magnetite nanoparticles were mixed with chitosan solution to form chitosan coated magnetite nanoparticles. The mixture was stirred at room temperature for 6 hours. The colloidal solution of $\mathrm{Fe}_{3} \mathrm{O}_{4}$ nanoparticles was centrifuged for $10 \mathrm{~min}$ (8,000 rpm). The chitosan coated magnetite nanoparticles was then washed with deionized water and dispersed in ethyl alcohol.

The coating duration was varied for 4, 6 and 8 hours. Then, the colloidal solution of chitosan coated magnetite nanoparticles was obtained after centrifuging for $10 \mathrm{~min}$ at $10000 \mathrm{rpm}$. Then, the particles were analyzed using AFM and FTIR studies. The effect of coating thickness with respect to coating time was also studied.

\subsection{Loading of drug and polymer to magnetite nanoparticles}

The drug sulphamethoxazole and the polymer chitosan were loaded to magnetite nanoparticles by adding $2 \mathrm{mg}$ of drug to $50 \mu \mathrm{l}$ of magnetite and then $950 \mu \mathrm{l}$ of ethanol was added to the solution. Then, the mixture was sonicated for 3 minutes. Again, the solution was sonicated for 10 minutes, while sonicating, the chitosan solution (1\% acetic acid) of 1 $\mathrm{ml}$ was added drop by drop to the mixture. The mixture was then stirred at room temperature until the ethanol evaporated. Then, $2 \mathrm{ml}$ of distilled water was added to the sediment powder. Then the colloidal solution of sulphamethoxazole/chitosan/magnetite nanoparticles was centrifuged for 15 minutes at $3000 \mathrm{rpm}$. The procedure was repeated for various concentrations of magnetite viz. $25 \mu \mathrm{l}$ and $15 \mu \mathrm{l}$.

\subsection{UV-Vis Analysis of Drug, Polymer loaded Magnetite Nanoparticles}

The drug, polymer loaded magnetite nanoparticles with different concentration of magnetite (viz.50, 25 and $15 \mu \mathrm{l}$ ) were analyzed using UV Spectroscopy. Initially, $100 \mu$ l of the drug, polymer loaded magnetite nanoparticle sample was taken in the cuvette and $3 \mathrm{ml}$ of ethanol was used as a solvent to characterize the sample. The absorbance values of all the three samples were noted.

\subsection{Characterization of Magnetite/Polymer Nanoparticles}

AFM was done to obtain the morphology of the magnetite/chitosan nanocomposite. The samples were analyzed using atomic force microscopy (Veeco CP II) in non-contact mode. The phase image for chitosan coated magnetite nanoparticles was recorded. This phase image reveals the information about coating over magnetite nanoparticles.

FTIR Spectroscopy is an appropriate technique to understand chemical adsorption or functionalization of $\mathrm{Fe}_{3} \mathrm{O}_{4}$ nanoparticles with polymers. The samples of magnetite and chitosan modified magnetite particles were ground with $\mathrm{KBr}$ and the mixture was compressed in to a pellet. FTIR (Thermo Nicolet, AVATAR 330, Mid IR,) was used with scanning range of $400-4000 \mathrm{~cm}^{-1}$ to characterize the chitosan coated- $\mathrm{Fe}_{3} \mathrm{O}_{4}$ nanoparticles.

\section{RESULTS AND DISCUSSION}

\subsection{Atomic Force Microscopy (AFM)}

The magnetite nanoparticles were analyzed by AFM with respect to their size and their size-distribution. Fig. 1 depicts the AFM images of the magnetite nanoparticles. The spherical shape of magnetic nanoparticles was obtained and its diameters were found to be in the range $5-50 \mathrm{~nm}$. The average particle size was found to be around $10 \mathrm{~nm}$.
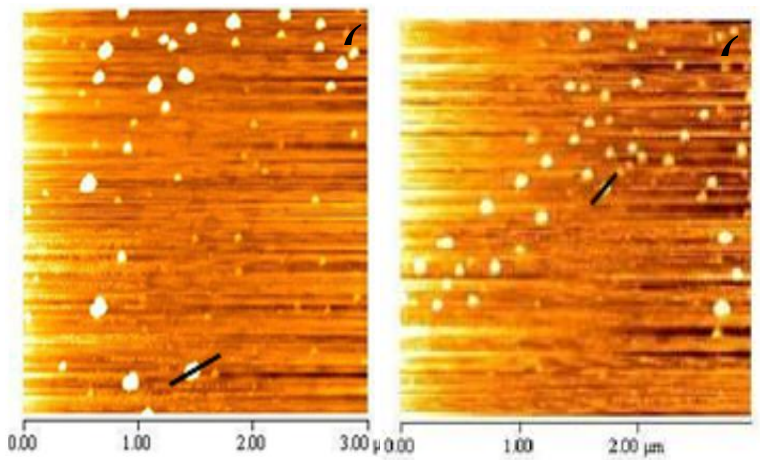

Figure 1 (a) \& (b) AFM images of magnetite nanoparticles.

Fig. 2 shows the AFM images of magnetite nanoparticles after chitosan surface modification. The coating duration was 4 hours. The particle size is found to be around $83 \mathrm{~nm}$ and the coating thickness is around $35 \mathrm{~nm}$.
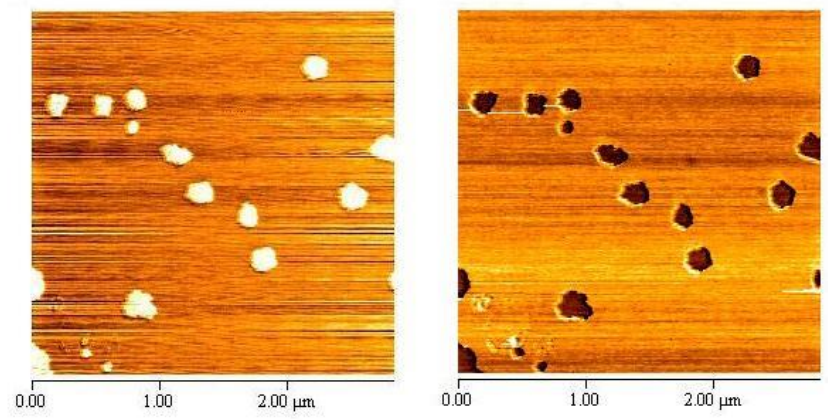

Figure 2 (a) Topography and (b) phase images of scan size $3 \mu \mathrm{m}$ of magnetite nanoparticles coated with chitosan for a coating duration of 4 hours

Fig. 3 shows the AFM images of the magnetite nanoparticles coated with chitosan for a coating duration of 6 hours. The magnetite-polymer core shell particle size was found to be around $14 \mathrm{~nm}$ and coating thickness of around 13 $\mathrm{nm}$ and $10 \mathrm{~nm}$. Fig. 4 depicts the phase image of magnetite nanoparticles coated with chitosan for a coating duration of 6 hours. 
The phase image confirmed that the chitosan was nicely coated over magnetite nanoparticles. The nanocomposite remained perfect spherical and most of the particles are uniform in size. The particle size is around $14 \mathrm{~nm}$ and the coating thickness is around $13 \mathrm{~nm}$ and $10 \mathrm{~nm}$.
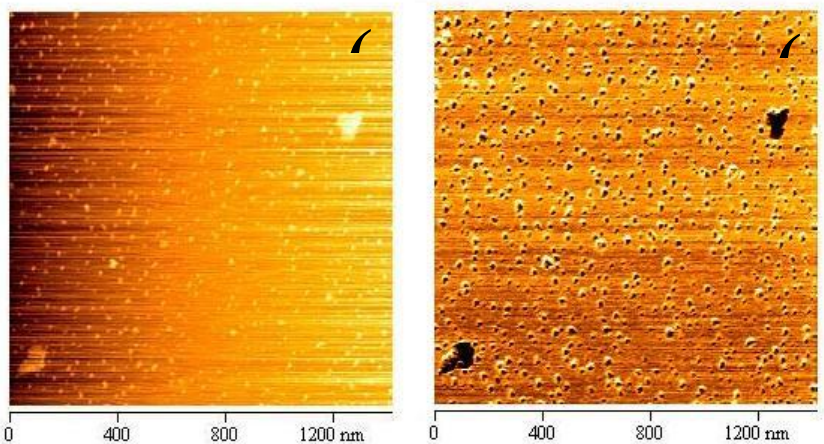

Figure 3 (a) Topography and (b) Phase images of scan size $1400 \mathrm{~nm}$ of the magnetite nanoparticles coated with chitosan for a coating duration of 6 hours
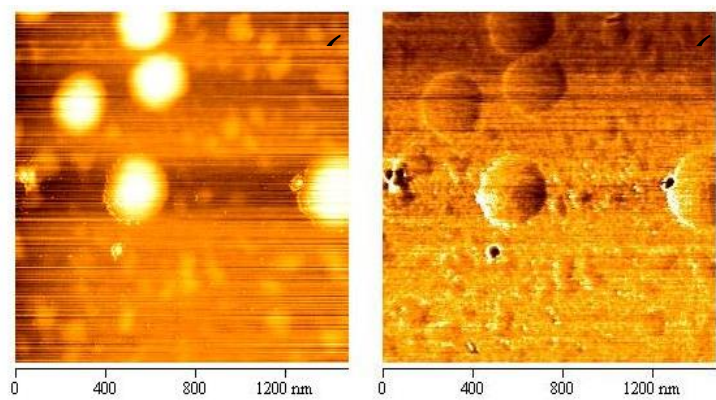

Figure 4 (a) Topography and (b) Phase images of scan size $1300 \mathrm{~nm}$ of drug, polymer loaded magnetite nanoparticles (concentration of magnetite is $50 \mu \mathrm{l}$ )

Fig. 5 presents the AFM image of sulphamethoxazole, magnetite nanoparticles coated with chitosan. The concentration of magnetite used was $50 \mu \mathrm{l}$. The particle size is $36.5 \mathrm{~nm}$ and the coating thickness is 17.8 $\mathrm{nm}$. The magnetite-polymer, drug core shell particle with particle size of $36.5 \mathrm{~nm}$ and coating thickness of $17.8 \mathrm{~nm}$ and $16.9 \mathrm{~nm}$. Fig. 6 shows the AFM image of sulphamethoxazole, chitosan coated magnetite nanoparticles for the concentration of $25 \mu \mathrm{l}$ of magnetite. The particle size is $18.4 \mathrm{~nm}$ and the coating thickness was $13.4 \mathrm{~nm}$. The magnetite-polymer core shell particle with particle size of $18.4 \mathrm{~nm}$ and coating thickness $13.4 \mathrm{~nm}$.
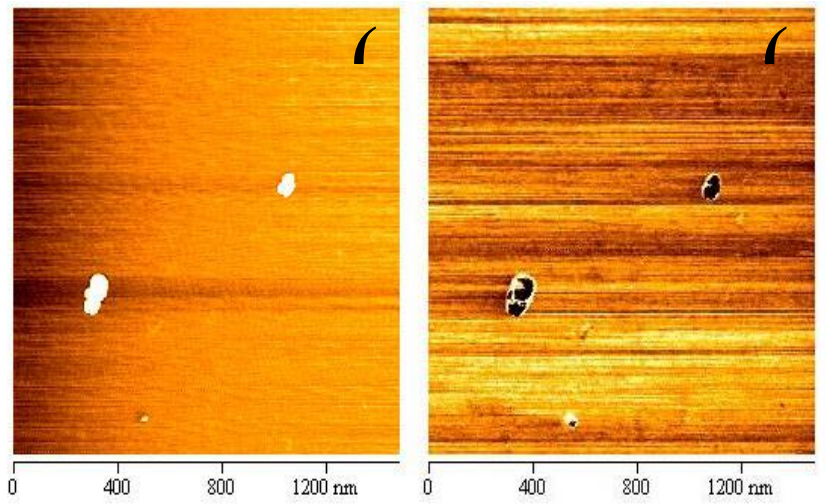

Figure 5 (a) Topography and (b) Phase images of scan size $1300 \mathrm{~nm}$ of drug, polymer loaded magnetite nanoparticles (concentration of magnetite is $25 \mu \mathrm{l}$ )

\subsection{Fourier Transform Infrared Spectroscopy (FTIR)}

The FTIR spectra of the magnetite nanoparticles coated with chitosan was shown in Fig. 6. The magnetite nanoparticles coated with chitosan possessed the characteristic peaks of chitosan. The absorption peak at 3434 $\mathrm{cm}^{-1}$ is corresponded to the hydroxyl $\left(\mathrm{OH}^{-}\right)$group and the peak at $1635 \mathrm{~cm}^{-1}$ is corresponded to the amine group $\left(-\mathrm{NH}_{2}\right)$. The absorption peak at $1015 \mathrm{~cm}^{-1}$ corrsponds to C-C and $\mathrm{C}-\mathrm{O}$ stretching modes of polysaccharides backbone, and the bending vibration of $-\mathrm{CH}_{2}$ group is at $1389 \mathrm{~cm}^{-1}$. The characteristic peak at $572 \mathrm{~cm}^{-1}$ corresponds to the magnetite nanoparticles which is also observed in the spectra of the magnetite nanoparticles coated with chitosan. Hence, this spectrum is confirmed that the magnetite nanoparticles are coated with chitosan

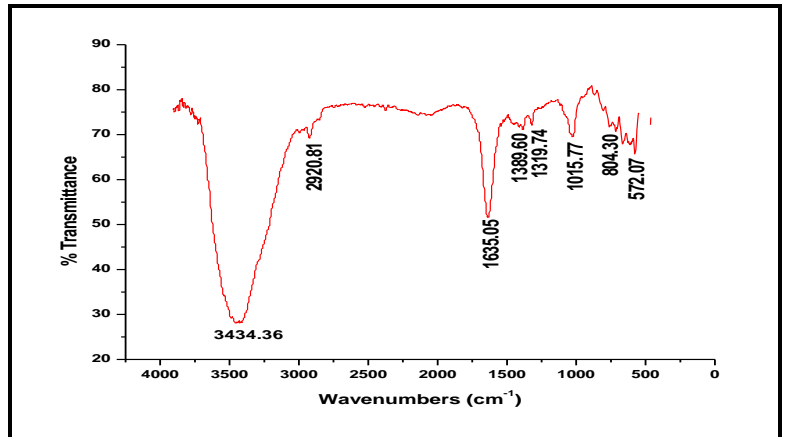

Figure 6 FTIR spectra of Chitosan coated Magnetite Nanoparticles

\subsection{UV-Vis spectroscopy}

Fig. 7 displays the UV-Vis spectra of the absorbance of polymer, drug loaded Magnetite at different concentrations of magnetite versus Wavelength. It is concluded that the absorbance increases as the concentration of magnetite increases. The encapsulation efficiency also increases as the concentration of magnetite increases. The encapsulation efficiency is $99.80 \%$ when the concentration of magnetite is $15 \mu 1$. However, the encapsulation efficiency is found to be $99.82 \%$ with the magnetite concentration of $25 \mu \mathrm{l}$. Therefore, it is concluded that the encapsulation efficiency is not significantly different in the presence of polymer.

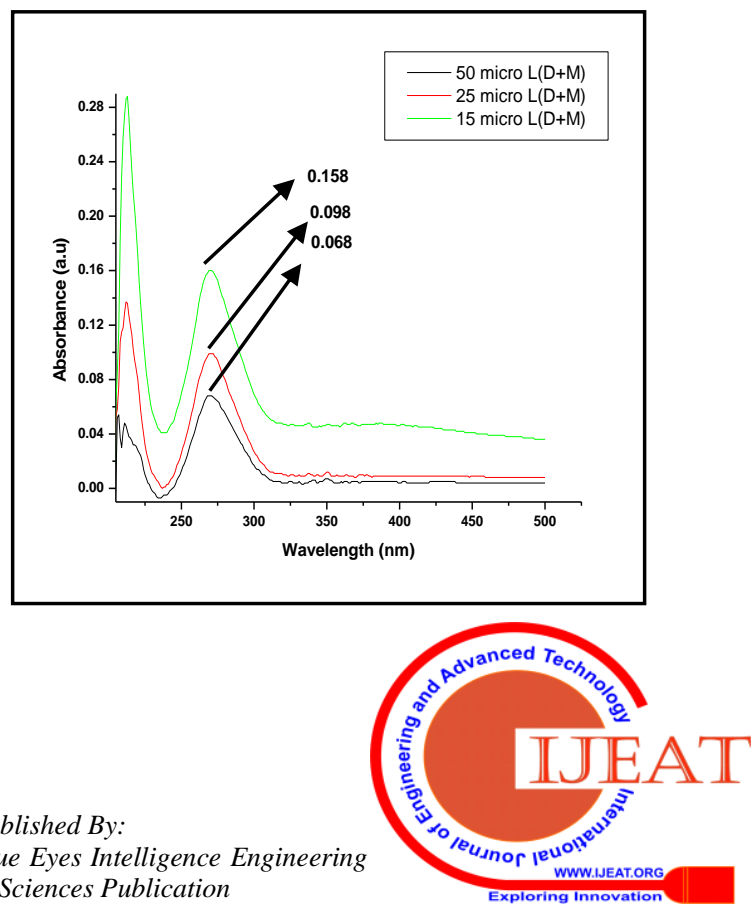




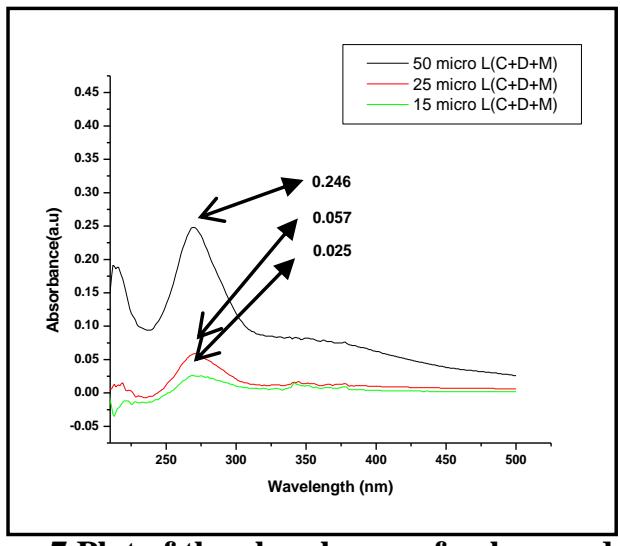

Figure 7 Plot of the absorbance of polymer, drug loaded Magnetite at different concentrations of Magnetite versus Wavelength

\section{CONCLUSION}

Atomic force microscopic (AFM) was used to characterize the magnetite nanoparticles as well as magnetite nanoparticles coated with chitosan. Ferric chloride and ferrous sulphate were used as the precursor for synthesizing the magnetite nanoparticles and analysed by AFM techniques in order to ascertain the size and shape using non-contact AFM. It was observed from the AFM analysis that the AFM images showed the spherical shape of the nanoparticles with 5-50 nm height. The average particle size was found to be 10 nm.

Chitosan was coated over the magnetite nanoparticles for varying duration of coating time. AFM phase images showed that individual $\mathrm{Fe}_{3} \mathrm{O}_{4}$ particles were nicely coated with the polymer. The polymeric nanoparticles have a core-shell structure with magnetite core and polymeric shell. The magnetite-polymer core shell has a typical particle size of around $14 \mathrm{~nm}$ and average coating thickness of around 11.5 nm.

The drug sulphamethoxazole was loaded over magnetite nanoparticles using solvent evaporation method. AFM phase images of the drug-loaded magnetite nanoparticles showed that the magnetite nanoparticles were coated with sulphamethoxazole. The drug, polymer loaded magnetite nanoparticles have a typical particle size of $36.5 \mathrm{~nm}$ and average coating thickness of around $17.4 \mathrm{~nm}$.

The drug loaded magnetite nanoparticles and drug/polymer loaded magnetite nanoparticles were also analyzed using UV-Vis spectroscopy. The encapsulation efficiency was calculated for the drug loaded magnetite nanoparticles and the polymer/drug loaded magnetite nanoparticles at different concentration of magnetite. It was found that the absorbance increases as the concentration of magnetite increases. It was found that the encapsulation efficiency is not significantly different in the presence of polymer.

\section{REFERENCES}

1. Lee N Hyeon T 2012 Designed synthesis of uniformly sized iron oxide nanoparticles for efficient magnetic resonance imaging contrast agents Chem. Soc. Rev. 412575 10.1039/C1CS15248C

2. Cardoso VF, Francesko A, Ribeiro C, Bañobre-López M, Martins P, Lanceros-Mendez S 2018 Advances in magnetic nanoparticles for biomedical applications Adv Healthc Mater. 7(5). doi: 10.1002/adhm.201700845

3. Joseph Raj X and Nishimura T 2014 Investigation of the Surface Potential on Iron Nanoparticles During the Corrosion by Atomic Force
Microscopy (AFM) and Kelvin Probe Force Microscopy (KFM) Int. J. Electrochem. Sci., 9 (2014) 2090 - 2100

4. Carvalho MD, Henriques F, Ferreir LP, Godinho M, Cruzb MM 2013 Iron oxide nanoparticles: the influence of synthesis method and size on composition and magnetic properties J. Solid State Chem 201 144-152. doi.org/10.1016/j.jssc.2013.02.024

5. Joseph Raj X 2018 Comparative Corrosion Behavior of Carbon Steel and High Chromium Steel Nanoparticles Research Journal of Nanoscience and Engineering Volume 2, Issue 3, 2018, PP 1-7 ISSN 2637-5591

6. Lazhen Shen, Yongsheng Qiao, Yong Guo, Shuangming Meng, Guochen Yang, Meixia Wu, Jianguo Zhao 2014 Facile co-precipitation synthesis of shape-controlled magnetite nanoparticles Ceramic international, 40, 1519-1524

7. Mohammad Reza Ghazanfari, Mehrdad Kashefi, Seyyedeh Fatemeh Shams, Mahmoud Reza Jaafari 2016 Perspective of Fe3O4 nanoparticles for biomedical applications, Biochemistry research journal, doi.org/10.1155/2016/7840161

8. Joseph Raj X 2018 A Comparative Study of the Corrosion Behavior of Mild Steel Nanoparticles in Natural Seawater and in $3.5 \% \mathrm{NaCl}$ Solution by AFM and KFM Techniques, J Fail. Anal. and Preven. 18 364-370 DOI 10.1007/s11668-018-0424-6

9. U. Jeong, X. Teng, Y. Wang, H. Yang, and Y. Xia 2007 Superparamagnetic colloids: controlled synthesis and niche applications, Advanced Materials, vol. 19, no. 1, pp. 33-60,

10. Adrienn J. Szalai, Nithyapriya Manivannan, George Kaptay, 2019 Super-paramagnetic magnetite nanoparticles obtained by different synthesis and separation methods stabilized by biocompatible coatings, Colloids and Surfaces A: Physicochemical and Engineering Aspects, $568,113-122$

11. J. K. Oh and J. M. Park 2011 Iron oxide-based superparamagnetic polymeric nanomaterials: design, preparation, and biomedical application, Progress in Polymer Science, vol. 36, no. 1, pp. 168- 189

12. Nhiem Tran, Thomas J. Webster 2010 Magnetic nanoparticles: biomedical applications and challenges, J. Mater. Chem. 20, 8760-8767

13. Daoush WM (2017) Co-Precipitation and Magnetic Properties of Magnetite Nanoparticles for Potential Biomedical Applications. J Nanomed Res 5(3): 00118. DOI: 10.15406/jnmr.2017.05.00118

14. Wei Wu, Zhaohui Wu, Taekyung Yu, Changzhong Jiang, Woo-Sik Kim 2015 Recent progress on magnetic iron oxide nanoparticles: synthesis, surface functional strategies and biomedical applications, Science and technology of advanced materials, 16, doi.org/10.1088/1468-6996/16/2/023501

15. Zhou L He B Zhang F 2012 Facile one-pot synthesis of iron oxide nanoparticles cross-linked magnetic poly(vinyl alcohol) gel beads for drug delivery ACS Appl. Mater. Interfaces 4192 10.1021/am201649b

16. Stefan Gustafsson, Andrea Fornara, Karolina Petersson Christer Johansson Mamoun Muhammed, Eva Olsson 2010 . Evolution of Structural and Magnetic Properties of Magnetite Nanoparticles for Biomedical Applications, Cryst. Growth Des. 10, 5, 2278-2284

\section{AUTHORS PROFILE}

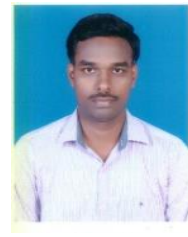

Mr.D.Balaji, received his M.Tech (Nanotechnology) in 2009 from Vellore Institute of Technology, Vellore. He is working as Assistant Professor in Department of Electronics and Communication Engineering, Vel Tech Rangarajan Dr.Sagunthala R\&D Institute of Science and Technology. His current research area focuses on Nanoelectronics, Drug delivery, Nanosensors and Nanoplasmonics.

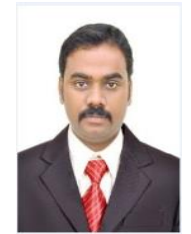

Mr.M.Vijay Albert William, received his M.E (Applied Electronics) from Anna University, Coimbatore. He is working as Assistant Professor in Department of Electronics and Communication Engineering, Vel Tech Rangarajan Dr.Sagunthala R\&D Institute of Science and Technology. His research area includes NDT, Nanomaterials and Embedded Systems. 


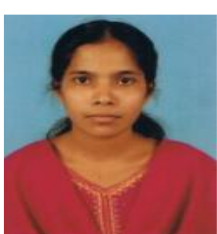

Ms.C.Sarala Rubi, received her B.Sc Physics from Manonmaniam Sundaranar University, Tirunelveli in 2006, and M.Sc Physics from the University of Bharathidhasan, Trichy in 2008. She was awarded M.Tech in 2010 by Anna University in Nano-Science and Technology. She is working as Assistant Professor in Department of Physics, Vel Tech Rangarajan Dr.Sagunthala R\&D Institute of Science and Technology. Her research interests include composites of the metal matrix, thermoelectric materials, and nano-materials. Six academic papers have been published in authoritative foreign journals. 